DEMOGRAPHIC RESEARCH

VOLUME 44, ARTICLE 36, PAGES 865-878

PUBLISHED 20 APRIL 2021

https://www.demographic-research.org/Volumes/Vol44/36/

DOI: 10.4054/DemRes.2021.44.36

Editorial

\title{
Editorial to the Special Issue on Demographic Data Visualization: Getting the point across - Reaching the potential of demographic data visualization
}

\section{Tim Riffe}

Nikola Sander

\section{Sebastian Klüsener}

This publication is part of the Special Collection "Data Visualization," organized by Guest Editors Tim Riffe, Sebastian Klüsener, and Nikola Sander.

(C) 2021 Tim Riffe, Nikola Sander \& Sebastian Klüsener.

This open-access work is published under the terms of the Creative Commons Attribution 3.0 Germany (CC BY 3.0 DE), which permits use, reproduction, and distribution in any medium, provided the original author(s) and source are given credit.

See https://creativecommons.org/licenses/by/3.0/de/legalcode. 


\section{Contents}

1 Introduction $\quad 866$

2 Why do we need good data visualization? 866

3 A brief history of data visualization in demography 868

$4 \quad$ Exploratory versus explanatory graphics $\quad 869$

$5 \quad$ Papers in the Special Collection 871

$6 \quad$ Best practice guidelines for creating effective data visualizations $\quad 873$

$\begin{array}{ll}\text { References } & 876\end{array}$ 


\title{
Editorial to the Special Issue on Demographic Data Visualization: Getting the point across - Reaching the potential of demographic data visualization
}

\author{
Tim Riffe ${ }^{1}$ \\ Nikola Sander ${ }^{2}$ \\ Sebastian Klüsener ${ }^{3}$
}

\begin{abstract}
BACKGROUND

Demography is full of data visualization challenges, such as age-period-cohort effects or life course trajectories. Innovative approaches to visualizing such complex data structures have been proposed from within and outside the discipline. However, demographic data visualizations presented in the scientific literature often fall short of the state-of-the-art.
\end{abstract}

\section{OBJECTIVE}

We discuss what makes a good data visualization and why it is worthwhile to strive for state-of-the-art visualization. We highlight the distinction between exploratory and explanatory graphics, and relate the seven papers that comprise the Demographic Research special collection on data visualization to the broader endeavor of data visualization in demography.

\section{CONTRIBUTION}

We suggest a set of best practice rules that are intended to provide general guidance for researchers attempting to produce meaningful and coherently designed figures from their data.

\footnotetext{
${ }^{1}$ Max Planck Institute for Demographic Research, Rostock, Germany. Email: Riffe@ demogr.mpg.de.

${ }^{2}$ Federal Institute for Population Research, Wiesbaden, Germany.

${ }^{3}$ Federal Institute for Population Research, Wiesbaden, Germany; Max Planck Institute for Demographic Research, Rostock, Germany; and Vytautas Magnus University, Kaunas, Lithuania.
} 


\section{Introduction}

Why do we visualize demographic data? In a scientific context, good visualizations enhance our understanding of the underlying data and grab the reader's attention without sacrificing truth for beauty. The seven papers that comprise the Demographic Research special collection on data visualization tackle this surprisingly challenging task. They present original visualizations that aim to facilitate the analysis, presentation, and dissemination of complex demographic data. The idea for this special collection emerged as part of the 2017 Rostock Retreat on Data Visualization, hosted by the Max Planck Institute for Demographic Research. ${ }^{4}$ This event was a practical exercise in data visualization excellence and experimentation, with reflection on the history and role of data visualization in demographic praxis and in other fields. The papers included in this special collection deal with topics discussed during the Rostock Retreat.

In this editorial, we begin with a brief overview of what motivates good data visualization, followed by a brief history of data visualization in the field of demography and a discussion of the distinction between exploratory and explanatory graphs. We then summarize the features of the papers that constitute this special collection, so that readers can choose the papers that most spark their interest. Lastly, we propose a set of best practice rules that are intended to provide general guidance for researchers attempting to produce meaningful and coherently designed figures from their data.

\section{Why do we need good data visualization?}

Data visualization refers to the graphical representation of information and data using visual elements composed into, for example, charts, graphs, or maps. Why are data visualizations so effective in communicating patterns and trends? Perhaps the most commonly cited argument for visualization is that the brain processes visual information (such as a bar chart) much faster than text (Healey 2018). Demographers are often tasked with understanding and summarizing large datasets. Good design takes advantage of our ability to decode visual patterns as information, optimizing the use of human senses to understand a (scientific) message. Visualizations can thus be employed to efficiently explore and discover patterns in demographic data, to help viewers gain a better understanding of magnitudes, intensities, durations, changes, and differences, and to communicate these to large audiences. Manuscript figures can also provide a more comprehensive overview of existing variation and exceptions to general patterns, which otherwise would require lengthy text descriptions that might still fall short of conveying

\footnotetext{
${ }^{4}$ See https://www.rostock-retreat.org/2017/.
} 
the same amount of information. In addition, figures such as flowcharts can provide a more intuitive understanding of methods (e.g., estimation procedures).

However, data visualization is used not only to convey information, but also as a research tool to explore. As such, visualization ought to accompany statistical analysis, both in deciding which dimensions of the data to look at in more detail, either with descriptive techniques or models, and which model results to report. Anscombe (1973) highlights the duality of statistical analysis and visualization, offering a quartet of small and structurally different datasets with almost identical descriptive statistics (see Figure 1). When plotted it becomes clear that the underlying data are structurally very different. This serves as a warning to fitting models without checking the data. Ideally, statistical analyses and graphics ought to give context to one another (Cook and Weisberg 1999).

Figure 1: Anscombe's quartet, showing four datasets with almost identical summary statistics that reveal very different distributions when graphed
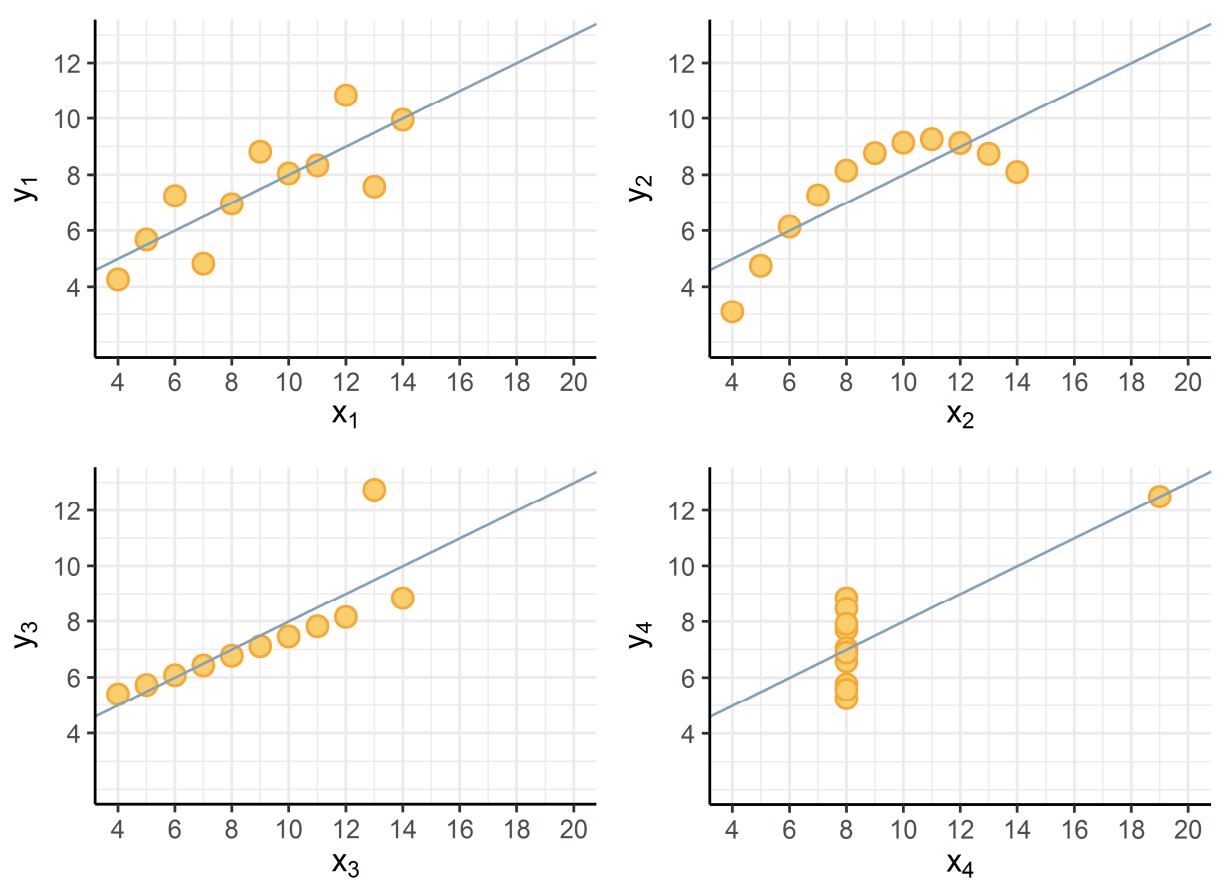

Source: Source: Dutta (2017), modified 


\section{A brief history of data visualization in demography}

In the field of demography, data visualization has a long history that goes back to at least the $19^{\text {th }}$ century. Figure 1 shows three classic examples of data visualization in demography: Perozzo's (1880) stereogram of the Swedish population, John Snow's Cholera map of London (Snow 1855), and Charles Joseph Minard's flow map of global migration (Minard 1861). In the late $19^{\text {th }}$ and early $20^{\text {th }}$ century, many publications in the Golden Age of Statistics featured marvelous maps and graphs (Friendly and Denis 2001). But producing such visuals in the analogue era was very labor-intensive and, unfortunately, widely ceased due to budget limitations during and after World War I. The early classics of demographic data visualization, such as the Lexis diagram of age-periodcohort patterns (Lexis 1875), did not lead to visualization becoming a primary endeavor in demography. Much to the contrary, the $20^{\text {th }}$ century saw a shift from graphics to tables and text, with visualizations being largely limited to bars and pies.

The boom in infographics and interactive data visualizations over the last few years has tempted demographers to rediscover the early classics for inspiration and to explore new techniques to visualize demographic phenomena. The digitization of scientific publication with a growing number of online-only journals provides more and more options for publishing visualizations in scientific papers. Moreover, the rise of social media allows scientists to share and showcase new visualizations and reach a broader audience outside the academic ivory tower. However, while the number of visualizations produced seems to increase exponentially, the share of high-quality visualizations seems far from increasing at a similar pace. One reason for this is that turning complex datasets into effective data visualizations for scholarly or wider reaching communication not only requires coding skills, but also visual design skills. So far, only few scientists master both skills equally well, and demographers are often rather leaning towards advanced coding skills. In terms of visual design skills, demographers can learn a lot from other disciplines with a stronger emphasis on visualizing data, such as data journalism, biology, geography, cartography, and architecture. 
Figure 2: Three classic examples of data visualization in demography by Perozzo (1881), Snow (1855), and Minard (1861)
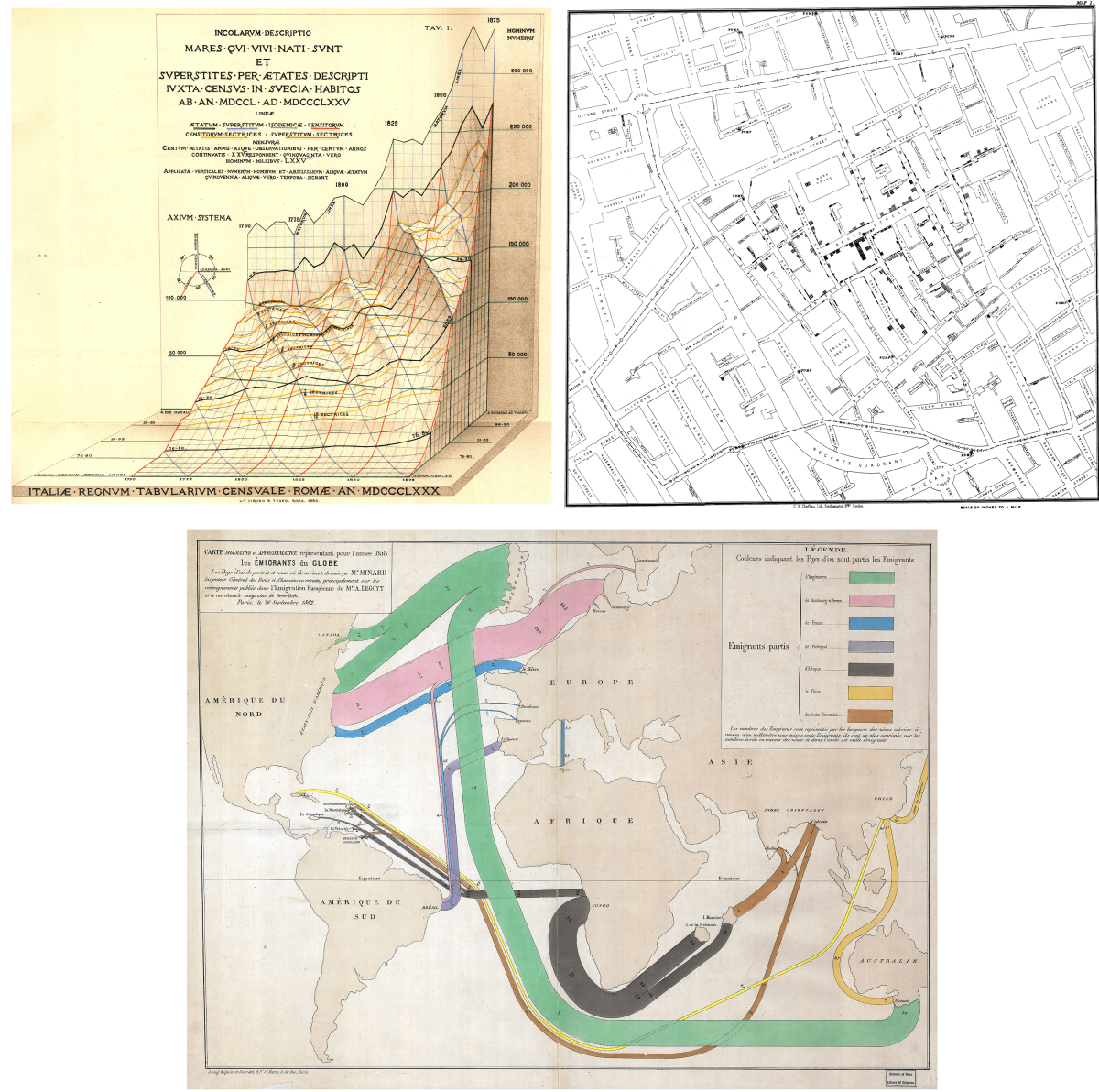

Note: All images are in the public domain.

\section{Exploratory versus explanatory graphics}

Visualizations are able to underline the multidimensionality of problems. In this sense, visualization is an exploratory tool that is part of the data analysis phase. Here, visuals 
are employed to dive deep into the complexity and detail of the dataset. As statistical graphics programs have grown to allow easy production of hundreds of figures with multiple layers of information, scientists are tempted to use these complex figures to communicate their findings. Whether intentional or not, the readers are confronted with too much or inefficiently delivered information, with the danger of even obfuscating the main message of a manuscript. To quote Moritz Stefaner: "Scientists do not seem to distinguish between analysis and communication. The last exploratory graph becomes explanatory." (Stefaner and Bertini 2014).

This can be avoided by making a clear distinction between exploratory and explanatory graphics:

Exploratory graphics are used in data analysis to reveal the multidimensional complexity of a dataset and find key patterns and trends. Since exploratory graphics are not usually meant to be shared with others, they have lower design requirements with less demand for a high "data-ink ratio" (Tufte 1983). ${ }^{5}$

Explanatory graphics are used to communicate patterns or information that are difficult to convey with text alone. Effective communication through this second type of visualization can only be achieved if the chart, graph, or map is designed in a coherent and clear way and really guides the reader to the main point, rather than confronting the reader with endless complexity and detail. As Garr Reynolds puts it, simplicity in the design of explanatory graphics can be achieved by remembering the "three fundamental principles: restrain, reduce, emphasize" (Acharya and Chellappan 2017).

In addition to exploratory graphics for data analysis and explanatory ones for communicating results, there is a kind of hybrid category that is gaining in significance and popularity with the rise of interactive data visualization tools. These hybrid visualizations usually show a curated dataset that has been distilled and facilitated to some extent to reduce noise and complexity. Such visualizations have a stronger freedomof-discovery aspect to the information presented than classic explanatory graphics. The intention is to allow some exploration on the reader's part, often using an interactive interface that lets the reader choose and constrain certain parameters, thereby discovering insights that might be new to both the reader and the creator of the visualization. Examples of hybrid visualizations of demographic data are the circular migration plot by Sander et al. (2014) (based on Krzywinski 2009) ${ }^{6}$, the Human Mortality Explorer based on Lexis plots (Schöley 2016) ${ }^{7}$, and the birth-flow chart by Riffe et al. (2019).

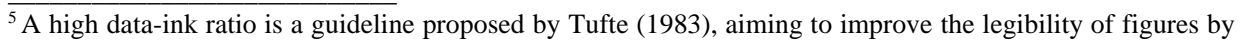
avoiding all graphical elements that are not essential to convey the data content. See also discussion below.

\footnotetext{
${ }^{6}$ Available at www.global-migration.info.

${ }^{7}$ Available at https://jschoeley.shinyapps.io/hmdexp/.
} 


\section{Papers in the Special Collection}

The seven papers that are included in this special collection cover both extensions of well-established demographic data representations such as the Lexis diagram, as well as illustrative applications of less widely used visualizations (e.g., ternary graphs, geofaceting, and ternary color compositions).

- Acosta and van Raalte (2019): APC curvature plots: Displaying nonlinear ageperiod-cohort patterns on Lexis plots

- Cimentada, Klüsener, and Riffe (2020): Exploring the demographic history of populations with enhanced Lexis surfaces

- Kashnitsky and Aburto (2019): Geofaceting: Aligning small multiples for regions in a spatially meaningful way

- Nowok (2020): A visual tool to explore the composition of international migration flows in EU countries, 1998-2015

- Pattaro, Vanderbloemen, and Minton (2020): Visualizing fertility trends for 45 countries using composite lattice plots

- $\quad$ Riffe and Aburto (2020): Lexis fields

- Schöley (2021): The centered ternary balance scheme: A technique to visualize surfaces of unbalanced three-part compositions

Acosta and van Raalte (2019) use a Lexis representation to visualize curvatures, the estimable nonlinear component of age, period, and cohort effects. The visualization technique captures and simplifies key features from several Lexis surfaces and displays them on a single surface, thereby enabling comparisons across surfaces. The authors apply APC curvature plots to show (a) the modal cohort of excess mortality from drugrelated causes by racial/ethnic group in the United States among the baby boomer generations, (b) the modal age of excess mortality in young adults, and (c) the modal age of fertility over cohorts and across populations.

Cimentada, Klüsener, and Riffe (2020) create enhanced Lexis surfaces in an attempt to overcome a limitation of conventional Lexis surfaces, which is the omission of information on population size when viewing rates. This limitation can lead to misinterpretations of mortality or other conditions that populations face over time. The authors demonstrate that adding the population size dimension to the Lexis surface enhances the analytical potential of standard Lexis surfaces. The paper is complemented by an interactive online web-application, which allows the reader to explore different dimensions of cohort mortality trends.

Kashnitsky and Aburto (2019) demonstrate how geofaceting preserves relative spatial location information by creating a "map of figures" in which each subfigure 
represents data for a specific location. The subfigures are situated in the plot according to their approximate relative location. In this visualization, readers can easily grasp whether neighboring regions share specific characteristics. Using data on young adult mortality in the 32 Mexican states from 1990 to 2015, and different graphical representations including ternary plot representations, the authors demonstrate how geofaceting supports the identification of spatial patterns in complex multidimensional phenomena.

Nowok (2020) uses ternary diagrams for analyzing international migration flow data to explore the different dimensions of migration over time and across countries. The paper is complemented by an interactive online web-application that shows immigration and emigration flows in the EU member states from 1998 to 2015. The data are grouped into three conventional categories (nationals of a reporting country, [other] EU nationals, and non-EU nationals) and presented in ternary diagrams.

Pattaro, Vanderbloemen, and Minton (2020) create composite lattice plots to visualize fertility data for multiple countries. These plots, for which the authors also developed an interactive online visualization tool, combine information from different visualization techniques of the Lexis surface, namely level plots and contour plots. By drawing on composite lattice plots, the paper explores changes in age-specific fertility rates (ASFRs) and the implied (period-based) cumulative cohort fertility rates (cumulative pseudo cohort fertility rates, CPCFRs) across countries and geographic regions.

Riffe and Aburto (2020) aim to extend the analytical potential of a Lexis surface representation by combining it with a vector field. Such Lexis fields allow to increase the dimensionality of patterns to be visualized. The paper exemplifies the use of Lexis fields by studying the relationship between life expectancy and the standard deviation of remaining lifespan over age and time. The example demonstrates how information layering on standard Lexis surfaces can reveal how relationships between multiple variables change over age and time.

Schöley (2021) proposes a method for using a ternary balance scheme for unbalanced data. The ternary color scheme is a visualization technique that encodes threedimensional compositions as a mixture of three primary colors. The adaptation to unbalanced data is achieved by reprojecting an unbalanced compositional data set around its center of location and visualizing the transformed data with a standard ternary color scheme. Data on the regional structure of the workforce in Europe is used to demonstrate the benefits of this technique when visualizing variation across unbalanced compositional surfaces. 


\section{Best practice guidelines for creating effective data visualizations}

The work of Edward Tufte (1983) has been very influential in establishing some heuristics for creating good data visualization. As many others, Tufte argues for what he calls "graphical integrity": that the visual representation of data should be as unbiased as possible and not, for example, over- or under-emphasize variance in the data. In addition, he offers some guidelines for elegant and clear graphics, with suggestions to aim for high "data density" and a high "data-ink ratio." That is, a high fraction of a figure's area should be devoted to the efficient representation of data rather than other plot chaff, such as space-greedy axes, distracting grid lines, 3D effects, or other unnecessary elements. Whether or not a plot element is important is certainly, at least to some degree, a subjective choice that depends on the purpose of the graphic and the target audience. If the audience is generally familiar with the visual encoding of a certain graphical type (e.g., a line graph, population pyramid, or a Lexis diagram), it is possible to reduce direct labeling and other nondata elements to a higher degree than if one is communicating with audiences that may not be familiar with this chart type. Tufte's guidelines are especially helpful for limiting the risk of losing the audience due to information overload, especially in oral presentations where audiences are given little time to understand the meaning of displayed graphics.

Inspiration for creating meaningful and coherent visualizations can also be found in the cartographic literature, which has a long-standing tradition in visualizing spatial information. As cartographers needed to produce maps of different scales, they were confronted early with the necessity to think of generalization, which in statistics finds strong analogy to data smoothing. When moving from maps of smaller areas to maps of whole regions, countries, or the world, cartographers have to deal with the issue of detail reduction. For example, plotting each of the tiny twists and turns of a river or coastline would result in a messy picture. Similarly, as demographers are confronted with data at ever-higher spatial and temporal resolution, the need for generalization and smoothing has gained importance. Here, data visualizations can also benefit from the recent progress in modeling methods to smooth data (e.g., Camarda 2012). For example, mortality surfaces or decompositions by cause of death can be very noisy and difficult to interpret unless they are smoothed (see, for example, the smoothed figures of Remund, Camarda, and Riffe 2018).

Color choices are critical decisions that make or break a visualization, because colors evoke different meanings, which in turn are influenced by culture and circumstances. Moreover, color scales should be used with care. Red and blue, for instance, may intuitively convey positive and negative values when used together, but this is obscured if positive is good and negative is bad, such as a choropleth depiction of changes in life expectancy. The use of color is also influenced by the type of data to be 
visualized: Color gradients are best for continuous data, while distinctive colors are best for categorical data. The color schemes suggested by Brewer (2003) are helpful in finding a color scheme that fits in a specific context. Color should always be an explicit choice and should be used strategically to guide the reader's or audience's eye. Lisa Charlotte Rost provides a friendly guide to colors in data visualization (Rost 2021).

Three-dimensional plots with three position scales (x, y, and $\mathrm{z}$ ) are a popular type of visualization, but they tend to be difficult to interpret. The key problem is the transformation of a 3D visualization space into a 2D space for printing. The human eye tries to invert this transformation, but fails at uniquely determining where a particular data point lies in the 3D space. For static images, it is thus recommended to show threedimensional data as contours or similar, or in a series of $2 \mathrm{D}$ plots. Interactive visualizations for the web allow the 3D space to be simulated, so that the reader can view the graphic from different angles, thus exploring the 3D space much more effectively than static graphics permit. Two examples of well-crafted interactive 3D visualizations are the Luminocity ${ }^{3 D}$ Urban density and dynamics explorer, ${ }^{8}$ which renders data binned on a spatial hexagonal tiling (Smith 2020), and the 3-D Lexis Pencil representation of categorical life trajectories (Francis, Fuller, and Pritchard 1998). Infotainment projects, such as the movies by 422 South $^{9}$ can also provide inspiration on how to deal with 3D data visualization.

We propose ten practical guidelines for creating great data visualizations for scientific manuscripts and presentations:

1. Use a standard chart type that most readers know how to interpret, unless there is no standard chart type that suits your data.

2. Choose a chart type that matches the data (i.e., line chart for time series, bar chart for categorical data).

3. Readers should be able to understand a figure without having to read the manuscript text. All necessary information should be provided in the figure and figure caption.

4. Figures should be designed so as to optimize clarity and readability. For instance, labels and text mark-up should be of legible size. Direct labeling is often more advantageous rather than side legends.

5. Embrace simplicity even when charting multidimensional data. Highlighting key relationships in complex data does not necessarily require a complex chart. For example, three-dimensional data does not necessarily need threedimensional charts.

\footnotetext{
${ }^{8}$ See https://luminocity3d.org/Population.html\#population_density_2011/11/51.5139/-0.0446.

${ }^{9}$ See http://422south.com/work/invisible-netherlands.
} 
6. Visual encoding, such as color, length, or area, should aim to enhance the legibility of the figure, and be deliberate, transparent, and justified. For example, the length of an element in a bar chart is much easier to decode by the reader than the area of a pie chart, and comparability holds up better as the number of categories increases. Ensure sufficient color contrast (e.g., avoid red on blue). Ensure that figures can still be read when printed in grayscale or viewed by colorblind readers.

7. If using categorizations, aim to make underlying data distributions transparent. In cases where presented results are subject to noteworthy statistical uncertainty, this should be visualized e.g., with different degrees of transparency, saturation gradients, or explicit depiction of bounds.

8. Figure axes (and projections in general) should not unintentionally distort relationships in the data. Distortions should be deliberate, transparent, and justified. For example, truncation of the y-axis in bar and line charts should be done with great caution as it can exaggerate differences in data.

9. Refrain from attempting to save time by using an exploratory graph for explanatory purposes. Instead allocate a greater fraction of the manuscript production time to figures, and recognize data visualization as an individual author contribution. Always aim to produce appealing figures that enhance understanding of the paper or presentation.

10. A well-made figure does not justify itself. Keep figures relevant to the purpose of the manuscript, and do not overwhelm the reader with unnecessary charts. 


\section{References}

Acharya, S. and Chellappan, S. (2017). Customizing data. Pro Tableau. Berkeley: Apress: 433-493.

Acosta, E. and van Raalte, A. (2019). APC curvature plots. Demographic Research 41(42): 1205-1234. doi:10.4054/DemRes.2019.41.42.

Anscombe, F.J. (1973). Graphs in statistical analysis. The American Statistician 27(1): 17-21. doi:10.1080/00031305.1973.10478966.

Brewer, C.A. (2003). A transition in improving maps: The ColorBrewer example. Cartography and Geographic Information Science 30(2): 159-162(4). doi:10.1559/152304003100011126.

Camarda, C.G. (2012). MortalitySmooth: An R package for smoothing Poisson counts with P-splines. Journal of Statistical Software 50(1): 1-24. doi:10.18637/jss. v050.i01.

Cimentada, J., Klüsener, S., and Riffe, T. (2020). Exploring the demographic history of populations with enhanced Lexis surfaces. Demographic Research 42(6): 149164. doi:10.4054/DemRes.2020.42.6.

Cook, R.D. and Weisberg, S. (1999). Graphs in statistical analysis: Is the medium the message? The American Statistician 53(1): 29-37. doi:10.1080/00031305.1999. 10474426.

Dutta, D. (2017). Anscombe's quartet. Available at: https://rpubs.com/debosruti 007/anscombeQuartet.

Francis, B., Fuller, M., and Pritchard, J. (1998). The use of visualization in the examination of categorical event histories. In: Blasius, J. and Greenacre, M. (eds.). Visualization of categorical data: 47-III. doi:10.1016/B978-012299045-8/50005$\mathrm{X}$.

Friendly, M. and Denis, D.J. (2001). Milestones in the history of thematic cartography, statistical graphics, and data visualization. http://www.datavis.ca/milestones.

Healey, K. (2018). Data visualization: A practical introduction. Princeton: Princeton University Press. https://socviz.co/.

Kashnitsky, I. and Aburto, J.M. (2019). Geofaceting: Aligning small-multiples for regions in a spatially meaningful way. Demographic Research 41(17): 477-490. doi:10.4054/DemRes.2019.41.17. 
Krzywinski, M., Jacqueline, S., Inanc, B., Joseph, C., Randy, G., Doug, H., Steven, J.J., and Marco, A.M. (2009). Circos: An information aesthetic for comparative genomics. Genome Research 19(9): 1639-1645. doi:10.1101/gr.092759.109.

Lexis, W.H.R.A. (1875). Einleitung in die Theorie der Bevölkerungsstatistik. Strassburg: KJ Trübner. doi:10.1515/9783111671987.

Minard, C.-J. (1861). Des tableaux graphiques et des cartes figuratives. Paris: Thunot. https://patrimoine.enpc.fr/document/ENPC01_Fol_10975.

Nowok, B. (2020). A visual tool to explore the composition of international migration flows in the EU countries, 1998-2015. Demographic Research 42(27): 763-776. doi:10.4054/DemRes.2020.42.27.

Pattaro, S., Vanderbloemen, L., and Minton, J. (2020). Visualizing fertility trends for 45 countries using composite lattice plots. Demographic Research 42(23): 689-712. doi:10.4054/DemRes.2020.42.23.

Perozzo, L. (1880). Della rappresentazione grafica di una collettività di individui nella successione del tempo, e in particolare dei diagrammi a tre coordinate. Annali di Statistica 2(12): 1-16. https://ebiblio.istat.it/digibib/Annali/TO00003841_Serie 02Vol12ed1880.pdf.

Perozzo, L. (1881). Stereogrammi demografici. Annali di Statistica 2(22): 1-20. https://ebiblio.istat.it/digibib/Annali/TO00003841_Serie02Vol22ed1881.pdf.

Remund, A., Camarda, C.G., and Riffe, T. (2018). A cause-of-death decomposition of young adult excess mortality. Demography 55(3): 957-978. doi:10.1007/s13524018-0680-9.

Riffe, T. and Aburto, J.M. (2020). Lexis fields. Demographic Research 42(24): 713-726. doi:10.4054/DemRes.2020.42.24.

Riffe, T., Barclay, K., Kluesener, S., and Bohk-Ewald, C. (2019). Boom, echo, pulse, flow: 385 years of Swedish Births. (MPIDR Working Paper WP-2019-002). doi:10.4054/MPIDR-WP-2019-002.

Rost, L.C. (2021). Your friendly guide to colors in data visualization. Datawrapper blog. 31/07/2018. Available at: https://blog.datawrapper.de/colorguide/.

Sander, N., Abel, G.J., Bauer, R., and Schmidt, J. (2014). Visualising migration flow data with circular plots. (Vienna Institute of Demography Working Papers 2/2014). https://www.oeaw.ac.at/fileadmin/subsites/Institute/VID/PDF/Publications/Work ing_Papers/WP2014_02.pdf. 
Schöley, J. (2016). The Human Mortality Explorer: An interactive online visualization of the Human Mortality Database. Paper presented at Population Association of America 2016 Annual Meeting, Washington, D.C.. https://paa.confex.com/ paa/2016/meetingapp.cgi/Paper/6383, Web application: https://jschoeley.shiny apps.io/hmdexp/.

Schöley, J. (2021). The centered ternary balance scheme: A technique to visualize surfaces of unbalanced three-part compositions. Demographic Research 44(19): 443-458. doi:10.4054/DemRes.2021.44.19.

Smith, D. (2020). Luminocity ${ }^{3 \mathrm{D}}$ Urban density and dynamics explorer. http://luminocity3d.org/indexRetina.html\#employ ment_density_2011/8/51.796/-1.074.

Snow, J. (1855). On the mode of transmission of cholera. London: Churchill. https://collections.nlm.nih.gov/ext/cholera/PDF/0050707.pdf.

Stefaner, M. and Bertini, E. (2014). The challenge of teaching visualization with Scott Murray and Andy Kirk. Datastories Podcast Episode 37. Available at: https://datastori.es/ds37-teaching-visualization-w-scott-murray-and-andy-kirk/.

Tufte, E.R. (1983). The visual display of quantitative information. Cheshire, CT: Graphics Press. 